\title{
Bilanz und Zukunft der Präsidentschaft im System des Rates der Europäischen Union
}

\author{
Daniela Kietz und Andreas Maurer*
}

Im ersten Halbjahr 2007 übernahm Deutschland den EU-Ratsvorsitz in einer kritischen Phase der europäischen Integrationsgeschichte. Nach den gescheiterten Referenden zum Vertrag über eine Verfassung für Europa, ${ }^{1}$ nach der langen und ergebnislosen Phase der Reflektion der europäischen Eliten über die zukünftige Gestaltung der Europäischen Union und vor dem Hintergrund der öffentlichen Kritik am ,Elitenprojekt Europa', musste der deutsche Vorsitz davon ausgehen, dass die Vorbehalte gegen die mit dem Verfassungsvertrag geplanten Integrationsschritte, gegen die europäischen Organe und gegen ihre Politiken erheblich zugenommen hatten. Gleichzeitig aber waren aus Sicht vieler Mitgliedstaaten die Erwartungen an die Präsidentschaft hoch, insbesondere durch die Wiederbelebung der Verhandlungen über den Verfassungsvertrag die Handlungsfähigkeit der Europäischen Union unter Beweis zu stellen.

Dem deutschen EU-Ratsvorsitz wurde in den europäischen Hauptstädten und Medien sowie seitens der Brüssler Institutionen eine weitgehend erfolgreiche Amtszeit bilanziert. ${ }^{2}$ Trotz der widrigen Umstände und vielschichtigen Erwartungshaltungen konnten in Grundsatzfragen der Integration (Vertragsreform), in strategisch und längerfristig angelegten Projekten (Energie- und Klimapolitik) sowie in einer Vielzahl laufender Legislativprojekte Konsens zwischen den EU-Mitgliedstaaten hergestellt und Kompromisse mit den anderen EU-Organen ausgehandelt werden. Gerade aufgrund dieser multifunktionalen Bandbreite der der Präsidentschaft abverlangten Aufgaben bietet sich das deutsche Beispiel an, über eine allgemeine Vorsitzbilanz hinaus, die Frage nach den wesentlichen, dossierübergreifenden Erfolgsfaktoren für EU-Ratspräsidentschaften zu analysieren und hieraus erste, vorsichtige Schlüsse für die im Lissabonner Vertrag vorgesehenen Reformen des Ratssystems zu ziehen.

Der folgende Beitrag umreißt zunächst die Funktionen und Aufgaben der EU-Ratspräsidentschaften sowie die Handlungsbeschränkungen unten denen diese agieren. Im Anschluss werden diejenigen Variablen herausgearbeitet, die den Weg für die zentrale Leistung des deutschen Vorsitzes ebneten, nämlich die erfolgreiche Vermittlung zahlreicher Verhandlungskompromisse. Der Beitrag schließt mit einem Blick auf den im Lissabonner Vertrag vorgesehenen Präsidenten des Europäischen Rates und die sich daraus ergebenden Fragen für die Organisation zukünftiger Ratspräsidentschaften.

* Dipl.-Pol. Daniela Kietz, Forschungsgruppe EU-Integration, Stiftung Wissenschaft und Politik, Berlin. Dr. Andreas Maurer, Leiter der Forschungsgruppe EU-Integration, Stiftung Wissenschaft und Politik, Berlin.

1 Im Folgenden Verfassungsvertrag.

2 Vgl. Daniela Kietz/Volker Perthes (Hrsg.): Handlungsspielräume einer EU-Ratspräsidentschaft. Eine Funktionsanalyse des deutschen Vorsitzes im ersten Halbjahr 2007, SWP-Studie, Nr. S 24/2007, Berlin. Centrum für angewandte Politikforschung (CAP): Bilanz der deutschen EU-Ratspräsidentschaft. Analyse und Bewertung, CAP Analyse 6/2007, München. Sebastian Kurpas/Henning Riecke: Is Europe Back on Track? Impetus from the German EU Presidency, CEPS Working Document 273/Juli 2007. 


\section{Funktionen und Aufgaben des EU-Ratsvorsitzes ${ }^{3}$}

\section{Der Ratsvorsitz als Manager der Ratsgeschäfte}

Die grundlegende Funktion des Ratsvorsitzes besteht in der effizienten Organisation der Ratsgeschäfte. Dazu gehört vor allem die zeitliche und inhaltliche Planung und Koordination der bis zu 4000 Sitzungen des Rates auf allen Ebenen (Arbeitsgruppen, interinstitutionelle Arbeitsgremien, Ausschuss der Ständigen Vertreter, Ministerräte etc.) während der sechsmonatigen Amtszeit. Die Arbeit der Ratsgremien muss zudem vom Ratsvorsitz, als Vertreter des Rates, in interinstitutionellen Verhandlungen mit dem Europäischen Parlament koordiniert werden, insbesondere bei Rechtsakten, die im Mitentscheidungs-, Zustimmungsund Haushaltsverfahren verhandelt werden. Mehr als bei den anderen Funktionen des Ratsvorsitzes, hängt die erfolgreiche Ausfüllung dieser Rolle von einer engen Kooperation des Vorsitzes mit dem Ratssekretariat ab, das vielfältige und lange eingeübte Koordinierungsressourcen vorhält und diese aufgrund der ständigen Präsenz in Brüssel und eingespielter Kooperationen mit vergleichbaren Einrichtungen im Europäischen Parlament zügig abrufen und in die ,Groupe de Coordination Interinstitutionnel ${ }^{6}$ - GCI einspeisen kann. ${ }^{4}$

\section{Der Ratsvorsitz als neutraler Makler von Kompromissen}

In seiner Funktion als Vermittler obliegt es dem Ratsvorsitz, Konsens herzustellen zwischen den Positionen der EU-Mitgliedstaaten bei den Verhandlungen im Rat und als Vertreter beziehungsweise Verhandlungsführer seitens des Rates bei den interinstitutionellen Verhandlungen zwischen Rat und Parlament sowie zwischen dem Rat und Drittstaaten im Bereich des auswärtigen Handelns. Neben den Vermittlungsbemühungen während der Sitzungen der entsprechenden Ratsgremien bedient sich die Präsidentschaft zur Konsensbildung auch bilateraler ,Beichtstuhlgespräche', um die Positionen der am Verhandlungsprozess beteiligten Akteure (Mitgliedstaaten, Europäisches Parlament, Drittstaaten) zu sondieren (wie der deutsche Vorsitz in der Vorbereitung der ,Berliner Erklärung' oder den Verhandlungen über den Verfassungsvertrag). Ein weiteres Instrument ist die Beauftragung von Expertengruppen oder die Bildung von Gremien (,Freunde der Präsidentschaft'), die sich aus Vertretern gleich gesinnter Mitgliedstaaten zusammensetzen und parallel zu den Verhandlungen in den Ratsgremien Kompromissvorschläge erarbeiten und damit die Verhandlungen beschleunigen beziehungsweise zur Deeskalation von Konflikten beitragen. Auch bei der Ausübung dieser Funktion, also der Beförderung und Herstellung von Verhandlungskompromissen, kann sich der Ratsvorsitz in zunehmendem Maße inhaltlich, taktisch und strategisch auf die Beamten des Ratssekretariats stützen.

\section{Strategische Steuerung durch den Ratsvorsitz}

Der Kern jener Aufgaben von EU-Ratspräsidentschaften, die in der angloamerikanischen Literatur unter dem Begriff ,Political Leadership“ und von uns unter dem der ,Strategischen Steuerung' zusammengefasst werden, besteht darin, tagesaktuelle Diskussionen unter den Mitgliedstaaten in einen größeren perspektivischen Zusammenhang mit den längerfristigen

3 Vgl. grundlegend Fiona Hayes-Renshaw/Helen Wallace: Taking Turns at the Wheel: The Presidency, in: Fiona Hayes-Renshaw/Helen Wallace: The Council of Ministers, 2. überarb. Aufl., Basingstoke 2006, S. 133-161; Ole Elgström: European Union Council Presidencies. A Comparative Perspective, London 2003; Jonas Tallberg: Leadership and Negotiation in the European Union, Cambridge 2006.

4 Vgl zum Ratssekretariat maßgeblich Fiona Hayes-Renshaw/Helen Wallace: Bureaucrats Organise and Advise: The Council Secretariat, in: Fiona Hayes-Renshaw/Helen Wallace: The Council of Ministers, 2. überarb. Aufl., Basingstoke 2006, S. 101-132. 
Herausforderungen für die Europäische Union zu stellen. Der Vorsitz muss die mitgliedstaatlichen Delegationen dazu mitunter anhalten, ihre kurzfristigen nationalen beziehungsweise wahltaktischen (Regierungs-)Interessen zugunsten langfristiger, unter allen EU-Mitgliedern abgestimmter europäischer Ziele hintanzustellen, wie dies der deutsche Vorsitz in der Klimapolitik versuchte.

\section{Der Ratsvorsitz als Akzentsetzer und Impulsgeber}

Die politische Agenda der Europäischen Union ist durch diverse, mehrjährige Planungsinstrumente auf lange Sicht vordefiniert. Es ist daher zentrale Aufgabe der Ratsvorsitze, fortlaufend für die Umsetzung und Anpassung dieser Agenden an den Stand ihrer Abarbeitung zu sorgen. Der verbleibende Raum zur Setzung eigener inhaltlicher Akzente, ist also deutlich geringer als gemeinhin angenommen. Dennoch gibt es Fälle, in denen es Ratspräsidentschaften gelingt, neue Projekte und Initiativen zur Lösung virulenter Probleme ,im Rahmen laufender Programme‘ zu lancieren (wie die Integration des Prümer Vertrages zur grenzüberschreitenden Kooperation in der Kriminalitätsbekämpfung in den EU-Rechtsrahmen unter deutschem Vorsitz) oder neue Ziele , außerhalb des Rahmens laufender Programme' zu formulieren (wie die Initiative der Bundeskanzlerin für einen transatlantischen Wirtschaftsraum). In der Regel versuchen alle Ratspräsidentschaften, durch derartige Initiativen der europäischen Politikagenda ihren eigenen Stempel aufzudrücken und im europäischen Einigungsprozess somit bleibende Spuren zu hinterlassen.

\section{Das Gesicht der EU: der Ratsvorsitz als externer Vertreter der EU in der Außenpolitik}

Auch die externe Vertretung der Europäischen Union im Bereich der Gemeinsamen Außen- und Sicherheitspolitik (GASP) obliegt dem Ratsvorsitz, zusammen mit dem Hohen Vertreter für die GASP und dem Kommissar für die Außenbeziehungen vertritt die Präsidentschaft die Union gegenüber Drittstaaten in allen diplomatischen Angelegenheiten (wie der deutsche Vorsitz im Rahmen des Nahostquartetts). Zudem fungiert sie - jenseits der Handels- und Entwicklungspolitik, in der die Kommission die Europäische Union vertritt als Sprachrohr der Europäischen Union in internationalen Organisationen und auf internationalen Konferenzen. In Verhandlungen über internationale Abkommen im Bereich der GASP und der polizeilichen und justiziellen Zusammenarbeit wird der Vorsitz vom Rat mit der Verhandlungsführung mandatiert (wie der deutsche Vorsitz in den Verhandlungen über das Fluggastdaten-Abkommen mit den USA).

\section{Vertretung der EU nach, innen': Mediatisierung und Inszenierung des Politikprozesses}

$\mathrm{Zu}$ den Aufgaben eines Ratsvorsitzes gehört es auch, die Handlungsfähigkeit der Union gegenüber den europäischen Bürgern, den Medien und den politischen Akteuren, vor allem im Heimatland zu demonstrieren. Analog zu entsprechenden Prozessen in der nationalen Politikarena wird hierzu die politische Agenda der Europäischen Union in der Regel auf wenige, eng umrissene Prioritäten und Konfliktlinien zugespitzt (wie Klima- und Energiefragen auf dem Frühjahrsgipfel während der deutschen Amtszeit), werden Verhandlungen und Verhandlungsergebnisse, insbesondere die Treffen des Europäischen Rates, medial und unter Nutzung einer ausgefeilten Dramaturgie und Symbolik inszeniert. Teil dieser gezielten Effekte sind die Vorausplanung inhaltlicher Spannungsbögen, die Wahl der Sitzungsorte für Sondergipfel und informelle Ministerratssitzungen, der Entwurf des Präsidentschaftslogos und die Formulierung eines griffigen Mottos. Gemeinsame, europaweite Herausforderungen und Probleme (illegale Einwanderung, Klimawandel, externe Krisensituationen) werden 
dramatisiert und die entsprechenden Lösungsansätze zeitlich so in Szene gesetzt, dass Beobachter den Eindruck bekommen, als identifizierten und lösten die Staats- und Regierungschefs ein akutes Problem , auf‘ dem Europäischen Rat.

\section{Handlungsbeschränkungen von Ratspräsidentschaften}

Bei der Erfüllung seiner verschiedenen Funktionen unterliegt der Ratsvorsitz einer Reihe von generellen strukturellen, materiellen und prozeduralen Beschränkungen, die seinen Handlungsspielraum eng begrenzen.

Erstens ist jede Ratspräsidentschaft zur Umsetzung der laufenden politischen Agenda der Europäischen Union verpflichtet. Die in Arbeits- und Rechtsetzungsprogrammen der EUOrgane festgeschriebenen Vorgaben schränken den Freiraum des Vorsitzes beträchtlich ein. Außerdem nehmen nicht vorhersehbare externe Ereignisse (Konflikte, Kriege, Terroranschläge, Naturkatastrophen und so weiter) einen großen Teil der Kapazität des Vorsitzes auf Kosten der ursprünglichen Planung in Anspruch.

Zweitens sind Ratspräsidentschaften auf einen Zeitraum von sechs Monaten befristet. Diese Spanne reicht meist nur aus, um entweder neue Projekte anzustoßen, laufende Verhandlungen voranzutreiben oder aber Projekte abzuschließen, die vorangegangene Präsidentschaften und andere EU-Akteure bereits maßgeblich befördert haben.

Drittens sind Ratspräsidentschaften nur ein Akteur unter vielen im europäischen Politikgestaltungsprozess. Sie agieren neben und mit der Kommission, dem Europäischen Parlament und anderen nationalen Delegationen im Rat, die gegebenenfalls eigene, vitale " und , institutionelle', teils schwer zu vereinbarende Interessen in einzelnen Dossiers geltend machen.

Und viertens unterliegen Ratspräsidentschaften innenpolitischen Beschränkungen. Sie müssen in der Planung und in der Durchführung ihres Vorsitzes laufend Ideen, Kritik und Begehrlichkeiten des Parlaments, der Fraktionen und Parteien, der subnationalen Gebietskörperschaften, aber auch der breiteren Öffentlichkeit berücksichtigen. Zudem sind interministerielle Koordinierungsprozesse für die Behandlung ressortübergreifender Dossiers auf die Funktionen des EU-Vorsitzes auszurichten, wobei das Konfliktpotenzial zwischen den Ministerien frühzeitig aufgefangen werden muss.

In diesen Handlungsbeschränkungen des Ratsvorsitzes liegt das Kernproblem bei der Bewertung seiner Arbeit: Welche Erfolge und Misserfolge lassen sich dem Wirken der Präsidentschaft zuschreiben? Ergebnisse, die als Errungenschaften oder Fehlschläge eines EUVorsitzes gefeiert beziehungsweise kritisiert werden, haben vielerlei Ursachen: Zum einen können die Erfolgs- beziehungsweise Misserfolgsfaktoren durchaus ,hausgemacht', das heißt durch das Verhalten der Regierung, die den EU-Vorsitz ausübt, selbst verursacht sein (zum Beispiel organisatorische, interministerielle Koordinierungs- und diplomatische Fähigkeiten). Zum anderen können aber externe Handlungsbeschränkungen außerhalb der Reichweite der Präsidentschaft dazu führen, dass Verhandlungen scheitern oder abgeschlossen werden (Vorarbeiten der letzten Präsidentschaft, Abschluss der Dossiers im Europäischen Parlament, auf die Europäische Union einwirkende Verhandlungen zwischen ,mächtigen " Drittstaaten und so weiter). Ein eindimensionaler Abgleich von Präsidentschaftsprogrammen und deren Ergebnissen oder das bloße Aufzählen von Projekten, die unter einer Ratspräsidentschaft vollendet wurden, sagen somit wenig über die Leistungsfähigkeit eines Vorsitzes aus.

Um diese Probleme bei der Bewertung von Ratspräsidentschaften zu bewältigen, greifen wir auf eine Methode zurück, die sich an der Frage orientiert, wie effizient der Vorsitz seine 
verschiedenen Funktionen erfüllt hat. ${ }^{5}$ Ausgehend von einem bereits erprobten, situativen Ansatz nehmen wir an, dass variierende Umfeldbedingungen von Verhandlungen unterschiedliche Anforderungen an den Ratsvorsitz in seinen Funktionen stellen. Werden die Anforderungen effizient erfüllt, wirkt sich dies positiv auf die Chancen für einen Verhandlungskompromiss aus. Entsprechend dieses Ansatzes nehmen effizient handelnde Präsidentschaften diejenigen Aufgaben war, die die gegebene Verhandlungssituation für ein erfolgreiches Abschließen oder Voranbringen der Dossiers von ihnen erfordert. ${ }^{6}$ Dies impliziert natürlich auch, dass eine Präsidentschaft die an sie gestellten Anforderungen und Funktionen sehr gut erfüllt haben kann und angesichts der diversen Handlungsbeschränkungen dennoch nicht das gewünschte Verhandlungsergebnis erreicht. Dennoch gilt: je besser ein Vorsitz seine Funktionen erfüllt hat, desto höher ist die Wahrscheinlichkeit eines Verhandlungsfortschritts.

Die zentrale Frage lautete damit: Inwieweit gelang es dem deutschen Vorsitz, seine Funktionen durch Wahrnehmung derjenigen Aufgaben zu erfüllen, die ihm unterschiedliche Verhandlungssituationen für ein erfolgreiches Voranbringen der Dossiers abforderten?

\section{Stärken und Schwächen des deutschen Vorsitzes}

Die Bilanz des deutschen Vorsitzes in der Erfüllung seiner Funktionen fällt in der Mehrheit der Dossiers durchweg positiv aus. ${ }^{7}$ Einzig mit Blick auf die langfristige, strategische Steuerung umstrittener Verhandlungsgegenstände ist die Bilanz eher gemischt. So entstand etwa im Kontext der Migrationspolitik der Eindruck, dass der Vorsitz die Behandlung verschiedener Aspekte legaler Wirtschaftsmigration - ein in Deutschland innenpolitisch sehr sensibles Thema - bewusst hinauszögerte oder aber dass Berlin wenig zur Schärfung und Klärung recht schwammiger, Konzeptdebatten` beitrug, da die Bundesregierung nicht in der Lage war, die eigene Position für alle sichtbar zurückzunehmen.

Der Präsidentschaft wird hingegen in den meisten Politikbereichen eine solide Managementleistung belegt. Die Arbeitsgremien wurden fokussiert und straff geleitet, auch wenn das Tempo kleinere Delegationen streckenweise überforderte (wie in der Migrations- oder Justizpolitik). Angesichts der über 50 Jahre entwickelten Expertise aller Fachressorts der Bundesregierung und der Landesregierungen, den Erfahrungen bei der Organisation vorangegangener Präsidentschaften und der umfassenden administrativen Ressourcen des größten EU-Staates durfte dies auch erwartet werden.

Die positive Leistungsbilanz des deutschen Vorsitzes tritt jedoch vor allem in der Vermittlung von Kompromissen und in der außergewöhnlich hohen Zahl an neuen, während seiner Amtszeit lancierten Projekten hervor. Der deutsche Vorsitz handelte als entschlossener, in manchen Fällen hart antreibender, aber doch weitgehend fairer Makler schwieriger Kompromisse. Diese Vermittlungsleistung stärkte die Stellung Deutschlands im Ministerrat und seinen (Mit-) Führungsanspruch ${ }^{8}$ in der Europäischen Union auch über die Ratspräsidentschaft hinaus. Neben der Glaubwürdigkeit als Makler, waren die Minimierung innerdeutscher Differenzen während der Amtszeit, eine klare Prioritätensetzung, eine überaus hohe und ressourcenaufwändige Anzahl bilateraler Konsultationen und informeller Gesprächsrunden, und vor allem das entschiedene, persönliche Engagement der Bundeskanzlerin und des Außenministers in hochsensiblen

5 Vgl. Adrian Schout/Sophie Vanhoonacker: Evaluating Presidencies of the Council of the EU: Revisiting Nice, in: Journal of Common Market Studies 5/2006, S. 1051-1077.

6 Vgl. für Details zur Vorgehensweise Kietz/Perthes: Handlungsspielräume einer Ratspräsidentschaft, 2007.

7 Vgl. hierzu die Einzelanalysen in: Kietz/Perthes: Handlungsspielräume einer EU-Ratspräsidentschaft, 2007.

8 Vgl. zum Konzept der (Mit-)Führung: Eckhard Lübkemeier: Führung ist wie Liebe. Warum Mit-Führung in Europa notwendig ist und wer sie leisten kann, SWP-Studie, Nr. S 30/2007, Berlin. Vgl. auch: Eckhard Lübkemeier: Europa braucht Führung, in: Frankfurter Allgemeine Zeitung vom 13. Dezember 2007. 
Bereichen wie der Energie- und Klimapolitik, der Nahostpolitik und der Revision des Verfassungsvertrages die Erfolgsvariablen. Diese sollen im Folgenden näher beleuchtet werden.

\section{Wegbereiter der Vermittlungserfolge}

Faires Makeln: Die von allen Akteuren wahrgenommene Fairness und Unparteilichkeit des Vorsitzes als Verhandlungsleiter sind grundlegend für die Formulierung eines konsensfähigen Kompromisses. Die Gratwanderung zwischen vermittelndem Vorsitz und der Vertretung nationaler Interessen war mit Blick auf die politischen und administrativen Ressourcen Deutschlands ein empfindliches Thema. Bei vielen Akteuren und Beobachtern bestand die Befürchtung, dass Deutschland als ,größter ‘ Mitgliedstaat seine (Eigen-)Interessen stark akzentuieren würde. Denn Erfahrungswerte zeigen, dass häufig kleine Mitgliedstaaten bessere Vermittler sind als große Mitgliedstaaten, denen es aufgrund stärkerer Partikularinteressen und innenpolitischer Koordinierungsanforderungen schwerer fällt, die notwendige Neutralität aufzubringen. Das Beispiel der britischen Ratspräsidentschaft im Jahre 2005 ist den meisten Beobachtern noch in guter Erinnerung. Aufgrund starker nationaler Interessen und den daraus resultierenden Beschränkungen, war die britische Regierung in wichtigen Verhandlungen (zum Beispiel über die finanzielle Vorausschau 2007-2013) nur bedingt handlungsfähig. ${ }^{9}$

Eine gute Erfüllung der Vermittlungsfunktion setzt voraus, dass die vorsitzstellende Regierung Konflikte zwischen dem nationalen Interesse, das sie als Delegation im Rat zu vertreten hat, und dem Unparteilichkeitsgebot der Ratspräsidentschaft vorab ausräumt. Um das Vertrauen der Mitgliedstaaten in seine Neutralität nicht zu gefährden, muss der Vorsitz dort, wo er ein starkes nationales Interesse geltend macht, dieses für alle Delegationen im Rat offenlegen und Vorbehalten der Mitgliedstaaten bereits vor Beginn seiner Amtszeit durch vertrauensbildende Maßnahmen begegnen. In jedem Fall muss sich der Vorsitz bemühen, trotz ausgeprägter Eigeninteressen mehrheitsfähige Kompromissvorschläge im Rat und gegebenenfalls gegenüber dem Parlament vorlegen zu können.

Zur Überraschung vieler Beobachter gelang es dem deutschen Vorsitz, trotz eigener Interessen im Rat akzeptable Kompromissvorschläge einzubringen, die die Meinungen einer großen Mehrheit der Delegationen beziehungsweise eine Konsenslinie widerspiegelten. In einigen Fällen wich der Vorsitz potenziellen Neutralitätskonflikten, die sich aufgrund ausgeprägter nationaler Präferenzen anbahnten, mittels bewährter Instrumente geschickt aus. Diese Konflikte wurden beispielsweise durch den Umweg über die EU-Institutionen umschifft. So wirkte die Bundesregierung in der Migrationspolitik schon unter der finnischen Ratspräsidentschaft 2006 darauf hin, dass die Kommission durch den Europäischen Rat beauftragt wurde, einen Bericht zur Migration über die östlichen EU-Außengrenzen vorzulegen. Auch in der Europäischen Nachbarschaftspolitik ließ Berlin eigene Ideen zur Weiterentwicklung dieses Politikfeldes in Kommissionspapiere einfließen, die unter finnischem Vorsitz autorisiert wurden und somit nicht direkt Deutschland zugeschrieben werden konnten. Beide Beispiele zeigen, dass eine erfolgsorientierte Ratspräsidentschaft, die ihre nationalen Eigeninteressen mit der Rolle des Vermittlers in Einklang bringen will, lange vor ihrer offiziellen Amtszeit beginnt. Auch das Beispiel der Madrider Gruppe der ,Freunde der Verfassung ' zeigt, wie der Vorsitz seine eigene Position und Prioritäten über Gruppen gleich gesinnter Mitgliedstaaten vertreten lassen kann, ohne die Glaubwürdigkeit als fairer Makler zu gefährden. ${ }^{10}$

9 Vgl. Kai Oppermann: Die britische Ratspräsidentschaft 2005: Zwischen europäischen Erwartungen und innenpolitischen Restriktionen, in: integration 1/2006, S. 23-37.

10 Neue Zürcher Zeitung: Die «EU-Verfassungs-Freunde» sammeln sich. Treffen der Befürworter des Entwurfs in Madrid, 27.01.2007. 
Wenn dennoch offen verfolgte nationale Interessen auf Unmut im Rat stießen (siehe zum Beispiel die deutsche Position zur Aufhebung der Sanktionen gegenüber Usbekistan), reagierte die Bundesregierung zügig und zeigte sich kompromissbereit, um jedem Glaubwürdigkeitsverlust vorzubeugen, der die Vermittlungsfähigkeit auch in anderen Dossiers beschädigt hätte.

Für die erfolgreiche Wahrnehmung der Impulsgeberfunktion im Verhältnis zur Vermittlungsfunktion scheint grundlegend zu sein, dass eine Initiative von den anderen Akteuren als effektive Antwort auf ein bestehendes, vom Ratsvorsitz identifiziertes europäisches Problem angesehen wird. Auch hier muss das vorsitzführende Land die Mitgliedstaaten im Rat möglichst vor Beginn der Amtszeit, mitunter über den Umweg dritter Spieler davon überzeugen, dass das von ihm angestrebte Projekt im europäischen und nicht nur im Eigeninteresse des Vorsitzlandes liegt. Erst dann können die anderen Delegationen das nötige Vertrauen in die Neutralität der Präsidentschaft aufbauen. In der wissenschaftlichen Literatur wird zumeist argumentiert, dass angesichts der existierenden EU-Programmplanung der Spielraum für Ratspräsidentschaften gering ist, völlig neue Projekte anzustoßen. Entgegen dieser Annahme ebneten die deutschen Akteure hinter den Kulissen des Rates und in Kooperation mit der Kommission bereits 2006 aktiv den Weg für eigene Initiativen und konnten gleich mehrere davon auf der europäischen Agenda platzieren. Die Überführung großer Teile des Vertrages von Prüm in den EU-Rechtsrahmen - eine maßgeblich deutsche Initiative zur Verbesserung des polizeilichen Datenaustausches - konnte der Vorsitz beispielsweise durch viel Überzeugungsarbeit den Mitgliedstaaten bereits vor Beginn seiner Amtszeit als ,europäisches' Projekt nahebringen.

Minimale innerstaatliche Differenzen: Konkurrierende Auffassungen auf innenpolitischer Ebene können die Handlungsfähigkeit der Ratspräsidentschaft auf europäischer Ebene beeinträchtigen und sie somit in der Erfüllung ihrer Funktionen behindern. Wenn Fachministerien gegenläufige Positionen zu einem Verhandlungsgegenstand vertreten, führt dies dazu, dass der EU-Vorsitz entsprechende Dossiers in den Ratsverhandlungen gar nicht oder nur mit großen Reibungsverlusten innerhalb der jeweiligen Regierung vorantreiben kann. Auch Differenzen zwischen den Partnern einer Regierungskoalition, zwischen verschiedenen innerstaatlichen Regierungsebenen oder zwischen Regierung und Parlament können sich negativ auf die Handlungsfähigkeit des Vorsitzes auswirken. In einigen Mitgliedstaaten wie Dänemark hat sich die Regel eingespielt, für den Zeitraum der Präsidentschaft eine politische Waffenruhe zwischen allen Parteien in Parlament und Regierung zu vereinbaren. Auch im Fall des EU-Ratsvorsitzes Sloweniens im ersten Halbjahr 2008 wurde eine solche Übereinkunft zwischen Regierung und Parlament fixiert. Damit wird gerade in Ländern, in denen das nationale Parlament ein großes Mitspracherecht in der Formulierung der Europapolitik hat und die Regierung an einer vergleichsweise kurzen Leine hält, der Tatsache Rechnung getragen, dass die Regierung in ihrem Amt als Ratsvorsitz über einen möglichst großen Handlungsspielraum zur Kompromissfindung verfügen muss.

Im Falle Deutschlands beeinträchtigten Differenzen auf nationaler Ebene die Vermittlungsleistung des Vorsitzes nur vereinzelt. Zumeist handelte es sich um interministerielle und parteipolitisch aufgeladene Konflikte der Regierungskoalition, die die Handlungsfähigkeit des Vorsitzes nur zeitweise beschränkten. Exemplarisch können hier die Auseinandersetzungen zwischen dem Bundesminister für Wirtschaft und Technologie (CSU) und dem Bundesminister für Umwelt, Naturschutz und Reaktorsicherheit (SPD) über die Begrenzung der Schadstoffemissionen für Kraftfahrzeuge angeführt werden. Dennoch drangen solche der Ressortautonomie geschuldeten Zuständigkeitskonflikte in den wenigsten Fällen an die für alle Akteure sicht- und in der Folge nutzbare Oberfläche. Offensichtlich waren die Berliner Ministerien intensiv bemüht, diese Konflikte zumindest für den Zeitraum der Ratspräsi- 
dentschaft zugunsten der Glaubwürdigkeit und Handlungsfähigkeit Deutschlands als neutralem Vermittler auf europäischer Ebene aus dem Weg zu räumen.

Klar definierte, gewichtete Prioritäten und konzentrierte Vermittlungsressourcen: Ein weiterer Faktor war für die erreichten Vermittlungserfolge besonders wichtig: die strategische Konzentration der Vermittlungsressourcen auf klar definierte Prioritäten der Ratspräsidentschaft.

Eine frühzeitige Definition der Prioritäten und Ziele ist Voraussetzung für deren Hierarchisierung und die darauf aufbauende effiziente Konzentration der Management- und Vermittlungsressourcen sowie für die Ausarbeitung strategisch-taktischer Vorlagen zur Umsetzung der Ziele. In diese Phase gehört auch die Abstimmung mit den Vorgänger- und Folgepräsidentschaften, die dazu dient, die Kohärenz und Kontinuität der europäischen Politik zu gewährleisten. Entsprechende Planungsgespräche müssen auch mit der Kommission geführt werden, da diese die Jahresgesetzgebungsprogramme verantwortet und die Arbeiten des Rates sich daran orientieren müssen. Kohärenzsicherungsprozesse müssen darüber hinaus mit Blick auf die interinstitutionellen Zeitpläne zu laufenden Verhandlungen und die vom Europäischen Rat autorisierten Mehrjahresprogramme in einigen Politikfeldern rechtzeitig eingeleitet und innerhalb der Administration des vorsitzführenden Landes in und zwischen den Fachressorts abgestimmt werden. Vorbereitung und Planung des deutschen Vorsitzes begannen auf nationaler Ebene nach dem Regierungswechsel im Herbst 2005. Diskontinuitäten gegenüber den Arbeiten der finnischen Präsidentschaft ergaben sich durch intensive Abstimmung kaum.

Unter deutschem Vorsitz hatte die Wiederbelebung der Verhandlungen über den Verfassungsvertrag oberste Priorität. Um deren Erfolg zu gewährleisten, ging der deutsche Vorsitz auch in anderen Verhandlungsfeldern sehr weitgehende Kompromisse gegenüber Ländern wie Polen (in den letztlich gescheiterten Verhandlungen mit Russland über ein neues Partnerschafts- und Kooperationsabkommen) und Frankreich (in den WTO- Verhandlungen im Rahmen der DOHA-Runde) ein, deren Zustimmung im Konflikt über den Verfassungsvertrag beziehungsweise den dann sogenannten Reformvertrag von zentraler Bedeutung war. Diese Form der Gewichtung von Kompromissen war nur möglich, weil sich die verhandlungsführenden Ministerien der Bundesregierung frühzeitig strategisch wie taktisch abstimmten und während der Verhandlungen über das Bundeskanzleramt koordiniert wurden (beziehungsweise sich koordinieren ließen).

Umfassende Konsultationen und Inklusivität: Politikfeldübergreifend trug außerdem die umfassende Nutzung bilateraler Konsultationen und informeller Gesprächsrunden auf allen Arbeitsebenen sowie bei Expertenseminaren maßgeblich zum Erfolg des deutschen Vorsitzes bei.

Seit den letzten Erweiterungsrunden klagen Delegationen im Rat immer wieder, dass die Entscheidungsfindung aufgrund der gewachsenen Anzahl der Vetospieler überaus schwierig geworden sei. Dies hat die Verhandlungsatmosphäre in den Ratsarbeitsgremien drastisch verändert. Die Zeiten, in denen sich eine kleine Gruppe Bekannter relativ offen und ausführlich austauschen konnte, gehören zumindest für die formalen EU-27-Kreise der Vergangenheit an. Die Auslagerung von Verhandlungen und Kompromissfindungsprozessen aus diesem engen formellen Rahmen ist daher nicht unbedingt Ausdruck der Geringschätzung des Ratssystems, sondern eine Reaktion auf die veränderte Verhandlungssituation im Rat. Insbesondere im Bereich der GASP sowie der polizeilichen und strafjustiziellen Zusammenarbeit wurde unter deutschem Vorsitz deutlich, dass angesichts der Anzahl von 27 Mitgliedstaaten die einstimmige Entscheidungsfindung im Rat die Leistungsfähigkeit des Vorsitzes stark beeinträchtigt. Gerade im Bereich der polizeilichen und strafjustiziellen Zusammenarbeit stan- 
den viele hochsensible Maßnahmen unter großem Termindruck zur Entscheidung an. Die deutschen Akteure vermittelten hier eine große Bandbreite von Kompromissen durch intensive Konsultationen nicht nur auf den unteren Arbeitsgruppenebenen des Rates, sondern auch direkt unter den Ministern und Staatssekretären. Positiv auf die Vermittlungstätigkeit wirkte sich in diesem Zusammenhang das relativ hohe Maß an Inklusivität, das heißt die Einbeziehung möglichst vieler - kleiner wie großer - Mitgliedstaaten im Vorfeld der Verhandlungen aus.

Der Erfolg der beobachteten Bilateralisierung der Verhandlungen beziehungsweise der Konsultation in kleinen, informellen Gruppen und der Verlagerung der Entscheidungsfindung auf die höchsten politischen Ebenen stellt im Umkehrschluss aber auch eine Bankrotterklärung für die derzeitigen formalen Entscheidungsmodalitäten innerhalb des Rats dar.

Gleichzeitig verweist dieser Erfolg auf die Grenzen des derzeit für den Ratsvorsitz geltenden Rotationssystems: Konsultationen in einem ähnlich großen Umfang, wie sie der deutsche Vorsitz in einer breiten Palette von Dossiers führte, um die schwerfälligen Entscheidungsprozesse zu beschleunigen, sind von Ratspräsidentschaften ,kleinerer ' Staaten mit weniger üppigen Ressourcen nur in viel begrenzterem Maße zu leisten. Dieselben Vorbehalte gelten für die Übertragbarkeit des organisations- und vermittlungsintensiven Vorgehens des Ratsvorsitzes in den Verhandlungen über die ,Berliner Erklärung ' und den Verfassungsvertrag. Den erst kürzlich beigetretenen Staaten mangelt es zudem an vergleichbaren Erfahrungen in der Verhandlungsführung und Kompromisserarbeitung. An diesen unterschiedlichen Voraussetzungen werden die Grenzen des für den Ratsvorsitz geltenden Rotationssystems bei gegebenen Entscheidungsregeln deutlich erkennbar.

Expertise und Engagement der zentralen Akteure: Das deutsche Beispiel zeigte nicht zuletzt, wie wichtig die über lange Zeit aufgebaute und ständig vorgehaltene Fachexpertise, gewachsene persönliche Beziehungen und vor allem das Engagement der zentralen Akteure des Vorsitzes für den Verhandlungsverlauf und -erfolg sind. Die deutschen Akteure konnten in den für die Vorbereitung, Planung und Durchführung der Präsidentschaft zentralen Gremien auf umfangreiche EU-Expertise und zu einem großen Teil auch auf die nunmehr fünfzigjährige Erfahrung mit der Organisation vergangener Präsidentschaften zurückgreifen. Hiervon profitierte der Vorsitz bei der Erfüllung seiner Management- und Steuerungsaufgaben, vor allem aber bei seiner Vermittlungstätigkeit.

Es muss zudem betont werden, dass die Kombination aus Expertise und persönlichem Einsatz - die sichtbare Entschlossenheit, einen Fortschritt in zentralen europäischen Verhandlungen erreichen zu wollen - nicht nur auf der Ebene der leitenden Ministerialbeamten, sondern auch auf der Ebene der Regierungschefs, der Minister und Staatssekretäre den Ausschlag für erfolgreiche Vermittlungen im Rat und gegenüber dem Europäischen Parlament geben können. Besonders augenfällig war dieser Erfolgsfaktor während der deutschen Amtszeit im Auftreten und im Engagement der Bundeskanzlerin und des Außenministers, aber auch im gesamten Politikfeld der Innen- und Justizpolitik zu beobachten. Entsprechend seiner starken Stellung auf der nationalen Ebene profilierten sich das Bundesinnenministerium und Minister Schäuble auch auf europäischer Ebene mit einem ambitionierten Programm und einer Reihe an Vermittlungserfolgen.

Die positiven Verhandlungsergebnisse in den Dossiers des Verfassungsvertrages, der Klima- und der Handelspolitik (transatlantischer Wirtschaftsraum) waren zu einem beträchtlichen Teil auf das direkte Engagement und die guten Beziehungen der Bundeskanzlerin zu verschiedenen Staats- und Regierungschefs zurückzuführen. Gerade in der Klimapolitik schien ihr politisches Gewicht und Geschick notwendig, um die Verknüpfung von G-8- und EU-Verhandlungen optimal auszunutzen. Das Vorgehen bei der Initiative zum transatlan- 
tischen Wirtschaftsraum zeigte zudem, dass eine Regierungschefin durch strategisch geplantes und taktisch durchkalkuliertes, persönliches Engagement die EU-Partner für ein neues Projekt zu gewinnen vermag. In diesem Fall gilt dies umso mehr, als die vom Kanzleramt koordinierte Initiative anfänglich nicht nur auf europäischer Ebene, sondern auch im deutschen Bundeswirtschaftsministerium auf nicht unbeträchtlichen Widerstand stieß.

Der Vergleich mit vorangegangenen Präsidentschaften größerer EU-Mitgliedstaaten man denke an die französische oder britische Präsidentschaft in den Jahren 2000 und 2005 zeigt, dass der starke persönliche Einsatz der Regierungschefin bis hin zur zentralen Leitung und Koordinierung etlicher Fachdossiers aus dem Kanzleramt heraus nicht einfach als gegeben angenommen werden kann. Wendet man den Blick nach vorn, auf zukünftige Präsidentschaften, entstehen unter diesem Gesichtspunkt Bedenken etwa hinsichtlich der Leistungsfähigkeit der tschechischen Ratspräsidentschaft. Es ist doch mehr als fraglich, wie viel Engagement die tschechische politische Elite, allen voran der Staatspräsident Klaus zeigen werden, um als EU-Ratsvorsitz eine überzeugende Agenda umzusetzen.

\section{Vermittlungserfolge zu Lasten von Transparenz?}

$\mathrm{Zu}$ den größten Vermittlungserfolgen des deutschen Vorsitzes zählen die ,Berliner Erklärung ' und das Mandat zur Aushandlung des Lissabonner Reformvertrages. Während die oben genannten Erfolgsvariablen zukünftigen Präsidentschaften als allgemein bewährte Praktiken dienen können, wirft insbesondere die Analyse dieser Dossiers jedoch auch die Frage auf, ob das vom deutschen Vorsitz gewählte Vorgehen diskretionärer Verhandlungen auf der Grundlage absichtlich herbeigeführter Asymmetrien im Informationsfluss für andere, vergleichbare Fälle Modell stehen kann. Denn diese Praxis geht zu Lasten der Transparenz der politischen Prozesse und Ergebnisse. Die hohe Vertraulichkeit der Verhandlungen hat sich zwar als geeignet erwiesen, in einer politisch hochsensiblen Situation Einvernehmen zwischen den 27 mitgliedstaatlichen Regierungen zu erzeugen. ${ }^{11}$ Gleichzeitig bedeutet der Erfolg dieses Vorgehens aber eine Absage an das ursprünglich, auf dem Europäischen Rat von Laeken im Dezember 2001 konsentierte Ziel, Grundfragen und -lagen der Integration nicht länger hinter verschlossenen Türen in kleinem Kreis zu beraten. ${ }^{12}$ Die deutsche Ratspräsidentschaft machte jedoch zu keinem Zeitpunkt den Versuch, einen in die Bürgergesellschaften hineinreichenden Konsens zu erzeugen. Der Nachteil dieses Vorgehens liegt auf der Hand: Bei erneuten Referenden über den Lissabonner Vertrag (in Irland ist dies Pflicht) besteht die Gefahr, dass sich die Bevölkerung eines oder mehrerer Länder wieder als Vetospieler der Umsetzung dieses Vertragswerkes in den Weg stellt.

Die deutsche Präsidentschaft hatte mit der Einigung auf ein Mandat zur Einberufung der Regierungskonferenz ein Ergebnis erreicht, dass alle Staaten auf ein politisches Ziel verpflichtete: die rasche Ausarbeitung eines runderneuerten Vertragswerks. ${ }^{13}$ Diese Einigung stellt ein beachtliches Ergebnis dar. Das Risiko, dem sich Deutschland und seine Partner aufgrund des Verfahrens ausgesetzt haben, ist allerdings hoch. Denn ein Scheitern des Lissabonner Vertrages wäre nicht nur grundsätzlich, sondern auch im Hinblick auf die Rolle Deutschlands in der Europäischen Union fatal: Das Reformmandat würde in diesem Fall als

11 Vgl. dazu auch Timo Goosmann: Die ,Berliner Erklärung “ - Dokument europäischer Identität oder pragmatischer Zwischenschritt zum Reformvertrag?, in: integration 3/2007, S. 251-263.

12 Siehe dazu auch Wolfgang Wessels und Anne Faber: Vom Verfassungskonvent zurück zur ,Methode Monnet“? Die Entstehung der, Road map“ zum EU-Reformvertrag unter deutscher Ratspräsidentschaft, in: integration 4/2007, S. 370-381.

13 Vgl. Rat der Europäischen Union: Schlussfolgerungen des Vorsitzes der Tagung des Europäischen Rates in Brüssel (21./22. Juni 2007), Dok. Nr. 11177/07, Anlage I: Entwurf des Mandats für die Regierungskonferenz, S. 15-30. 
,zu eng ' verurteilt und der ,Übermacht` Deutschlands zugerechnet. Damit würde das Scheitern des Lissabonner Vertrages mit der Rolle Berlins in der Europapolitik allgemein in Verbindung gebracht. Deutschlands Führungsanspruch in der Europäischen Union wäre damit schwer beschädigt.

Welche Lehren können aus dem praktizierten Verfahren und dem hierbei ermittelten Funktionsprofil der deutschen Ratspräsidentschaft beim Blick auf das Dossier des Verfassungsvertrages für künftige Vorsitze gezogen werden? Offensichtlich ist eine alle Seiten befriedigende Vermittlungsfunktion in institutionellen Krisen nur zum Preis extremer Transparenz (zum Beispiel in der Konventsmethode) oder starker Intransparenz zu haben. Wählt man die erste Variante - der Lissabonner Vertrag erlaubt dieses Verfahren explizit (Artikel 48 EUV) -, ist eine erfolgreiche Vermittlung verschiedener Interessenlagen leichter, wenn diese Funktion gemeinsam mit Akteuren gestaltet wird, die keinen mitgliedstaatlichen Weisungen unterliegen, sondern glaubhaft im gemeinsamen Interesse aller Beteiligten agieren können. Greifen die Mitgliedstaaten dagegen auf die zuletzt praktizierte Variante der Geheimverhandlungen zurück, ist das Risiko groß, dass nicht nur einzelne Akteure, sondern auch die Ratspräsidentschaft die ,Bodenhaftung verlieren und an den Interessen und Bedürfnissen der Bürgergesellschaften vorbei handeln. Der Preis hierfür wird spätestens bei Wahlen zu zahlen sein, wenn sich Bürger immer weiter von etablierten Parteien abwenden und ihre Stimme Populisten und Extremisten geben.

\section{Ausblick: Fragezeichen des Lissabonner Vertrages}

Die Analyse wäre unvollständig, wenn sie die Funktionsbilanz nicht mit den im Lissabonner Vertrag vorgesehenen Änderungen im Ratssystem in Bezug setzen würde. Denn aller Voraussicht nach ändern sich mit Inkrafttreten des Lissabonner Vertrags Mitte 2009 die Grundregeln der Vorsitzführung im Ratssystem: Ein auf zweieinhalb Jahre gewählter Präsident sitzt dann dem Europäischen Rat vor und ein auf fünf Jahre von Rat und Parlament ernannter „Hoher Vertreter der Union für die Außen- und Sicherheitspolitik“ (HVU-ASP) übernimmt den ständigen Vorsitz des Rates Außenbeziehungen. Das Rotationsprinzip wird in Zukunft auf die übrigen Fachministerräte beschränkt, wobei eine Verstetigung der 2007 erstmals getesteten, achtzehnmonatigen, Teampräsidentschaften angestrebt ist.

An einer grundlegenden Reform des Systems aus Europäischem Rat und Ministerrat wird seit Ende der 1990er Jahre gearbeitet, um die organisatorischen Herausforderungen der 2004 und 2007 erfolgten Erweiterungen aufzufangen. Oberstes Ziel ist hierbei die Verbesserung der Handlungsfähigkeit des Ratssystems nach innen - im Hinblick auf seine Binnenstruktur der Fachratskoordination sowie auf die Zusammenarbeit des Rates mit dem Europäischen Parlament und der Kommission - und nach außen gewesen - im Hinblick auf die Verbesserung der Kohärenz, Identifizierbarkeit und Durchsetzungsfähigkeit in der Außen- und Sicherheitspolitik. ${ }^{14}$ Gemäß den von allen 27 EU-Staaten bereits 2001 in der Erklärung von Laeken aufgestellten Anforderungen einer handlungsfähigeren, demokratischeren und transparenteren Europäischen Union wird schließlich auch die Verbesserung der Impulsgebungsund Steuerungsfähigkeiten des Europäischen Rates und die Stärkung der Funktionen des Vorsitzes im Rat und im Europäischen Rat zur Koordinierung, Steuerung und Führung des Rates angestrebt. Diese Reformen werden sich nachhaltig auf die Handlungsmöglichkeiten ,nationaler' Ratsvorsitze auswirken.

14 Vgl. ausführlicher Andreas Maurer: Auf dem Weg zur Staatenkammer. Die Reform des Entscheidungs- und Koordinationssystems im Ministerrat der EU, SWP-Studie, Nr. 6/2003, Berlin. 


\section{Die Vorsitzfunktion in der Außenpolitik}

Seit Jahren unterstreichen politische Akteure und Beobachter der Europapolitik die Notwendigkeit verstärkter Kontinuität und Kohärenz in der EU-Außenpolitik. Der im Verfassungsvertrag vorgesehene und vom Lissabonner Vertrag in seinen Funktionen bestätigte HVU-ASP (Artikel 18 EUV) soll hier Abhilfe schaffen. Durch seine Doppelhutfunktion in Rat und Kommission wird er den Vorsitz in der neuen Ratsformation für Auswärtige Angelegenheiten ausüben und ab 2009 gleichzeitig als Vizepräsident der Kommission fungieren. ${ }^{15}$ Hiermit wird die Koordinierung und Durchführung der EU-Außenbeziehungen einschließlich der zivilen und militärischen Aspekte europäischer Missionen in Absprache mit den nationalen Außenministern im Rat erstmals auf ein Amt und eine Person konzentriert. Die Formulierung der gemeinsamen europäischen Außenpolitik fällt dem Hohen Vertreter ebenso zu wie eine internationale Repräsentationsfunktion. Mit der Koordinierungsfunktion in Rat und Kommission kommt ihm damit ein ungewöhnlich großer Aufgabenbereich und besondere Verantwortung in einem institutionellen Spannungsfeld zu. Er wird nicht nur vor die Herausforderung verschiedener Verfahren, Kompetenz- und Legitimationsquellen, sondern auch in ein strukturelles Spannungsverhältnis zu anderen Akteuren gestellt. Denn neben den nationalen Außenministern werden der Präsident der Kommission und der Präsident des Europäischen Rates im außenpolitischen Bereich agieren.

Die Schaffung des neuen Außenamtes der Europäischen Union stellt zudem die 2002 vereinbarte Reduzierung der Ratsformationen infrage. Denn sachlich nachvollziehbar ist die Wahrnehmung der Vorsitzrolle als ,Ratsmitglied' bei gleichzeitiger Wahrnehmung der Rolle des Kommissionsamtes bisher nur im außen- und sicherheitspolitischen Teil des AuBenrates. Eine entsprechende Personalunion für die gegenwärtig auch in dieser Ratsformation behandelten Tagesordnungspunkte zur Außenhandelspolitik und Entwicklungszusammenarbeit würde dagegen die historisch gewachsene Eigenständigkeit und das Profil der Kommission sowie die Wahl der Kommissare in diesen Bereichen grundsätzlich infrage stellen. Organisatorisch könnte die Schaffung des neuen EU-Außenamts damit die Verknüpfung und langfristige Zusammenführung von mindestens fünf Generaldirektionen in der Kommission mit zwei Direktionen im Generalsekretariat des Rates, den Wegfall wichtiger Kommissionsportfolios und damit eben auch prominenter Ernennungsmöglichkeiten für die Mitgliedstaaten bedeuten. Es wäre daher angebracht, die Einrichtung des neuen Außenamtes im Lichte der damit einhergehenden Struktur der Ratsformationen und -vorsitze auf allen Arbeitsebenen zu überprüfen. Sollte sich der HVU-ASP künftig vor allem auf das enge Themenfeld der GASP und Europäischen Sicherheits- und Verteidigungspolitik konzentrieren, dann müsste zwangsläufig darüber nachgedacht werden, die 2002 abgeschaffte Ratsformation ,Entwicklungszusammenarbeit" wiederherzustellen sowie die Neugründung eines AuBenhandelsrates zu erwägen, um die Funktionen der Kommission und ihre Rechenschaftspflicht gegenüber dem Europäischen Parlament aufrechtzuerhalten.

Auf jeden Fall kommt dem HVU-ASP in seiner Doppelhutfunktion nicht nur die Aufgabe zu, die Kohärenz und Konsistenz des auswärtigen Handelns der Union zu befördern, sondern auch für einen Ausgleich zwischen der zwischenstaatlichen Logik der GASP und der supranationalen Logik der Gemeinsamen Handelspolitik (GHP) zu sorgen und dabei zu gewährleisten, ,dass die zwischenstaatliche Logik der GASP die GHP nicht negativ beeinflusst". 16

15 Innerhalb der Kommission wird der HVU-ASP mit den Zuständigkeiten der bisherigen Kommissarin für die Außenbeziehungen der Europäischen Union betraut sein. 


\section{Potenzielle Macht des neuen Präsidenten: ein gestärkter Europäischer Rat}

Der Präsident des Europäischen Rates soll ab 2009 für eine Zeitspanne von jeweils zweieinhalb Jahren die Beratungen des Europäischen Rates leiten und diesem - relativ - neuen Organ der Europäischen Union vorsitzen. In dieser Funktion ist er auch für die Vorbereitung und Kontinuitätssicherung der Gipfeltreffen zuständig, wobei er mit dem Präsidenten der Europäischen Kommission kooperieren und sich auf die Vorarbeiten des Rates ,Allgemeine Angelegenheiten' stützen soll. Darüber hinaus ist der Präsident im Lissabonner Vertrag aufgerufen, ,,auf seiner Ebene und in seiner Eigenschaft, unbeschadet der Befugnisse des Hohen Vertreters der Union für Außen- und Sicherheitspolitik, die Außenvertretung der Union in Angelegenheiten der Gemeinsamen Außen- und Sicherheitspolitik" wahrzunehmen (Artikel 15 Absatz 6 EUV).

Diese Aufgabenbeschreibung ist wenig aussagekräftig. Der um diesen Vertragsartikel geworfene Schleier lichtet sich erst beim Blick auf die im Lissabonner Vertrag angeführten Handlungsermächtigungen des Europäischen Rates. Denn erst diese geben das sachliche Aufgabenspektrum des Vorsitzenden des Europäischen Rates wieder. Nach Artikel 15 Absatz 1 EUV gibt der Europäische Rat nun ,der Union die für ihre Entwicklung erforderlichen Impulse und legt die allgemeinen politischen Zielvorstellungen und Prioritäten fest“. Diese Rollendefinition lehnt sich an die gültige Funktionszuschreibung aus dem Vertrag von Nizza an. Gleichwohl gehen die im Lissabonner Vertrag einzeln aufgeführten Aufgaben des Europäischen Rates weit über die in Artikel 15 Absatz 1 EUV definierte Rolle hinaus. Der Europäische Rat wird künftig über Beschlussfassungs-, Benennungs-, Wahl- und Abberufungsrechte verfügen:

Beschlussfassungsrechte institutioneller Art erhält der Europäische Rat im Hinblick auf die Zustimmung zum Vorschlag über die Zusammensetzung des Europäischen Parlaments, zur Festlegung der Zusammensetzung der einzelnen Ratsformationen, zur Festlegung des Rotationsprinzips in den Ratsformationen, zur Verlängerung der Ausnahmebestimmungen im Protokoll über die Vertretung der Bürger im Europäischen Parlament und die Stimmengewichtung im Rat, zur Überführung besonderer Rechtsetzungsverfahren in das normale Gesetzgebungsverfahren, zur Überführung der Einstimmigkeitspflicht im Rat in den Entscheidungsmodus der qualifizierten Mehrheit, zur Festlegung der paritätischen Rotation innerhalb der Kommission, und zur Prüfung der vorgeschlagenen Änderungen zu den Verträgen und der Festlegung eines Mandats für neuerliche Regierungskonferenzen.

Politikbereichsspezifische Beschlussfassungsrechte überträgt der Vertrag dem Europäischen Rat zur Verabschiedung allgemeiner GASP-Beschlüsse, zur Überführung des Entscheidungsmodus des Rates in der GASP von der Einstimmigkeit in die qualifizierte Mehrheit, zur Feststellung, dass die gemeinsame Verteidigungspolitik zu einer gemeinsamen Verteidigung führt, zur Festlegung von Leitlinien hinsichtlich der Abkommen der Union mit einem Mitgliedstaat, der aus der Union auszutreten beabsichtigt, zur Fristverlängerung im Hinblick auf die Anwendung der EU-Verträge in einem Mitgliedstaat, der aus der Union austritt, zur Verabschiedung von Schlussfolgerungen zu den Grundzügen der Wirtschaftspolitik der Mitgliedstaaten und der Union, zur Verabschiedung von Schlussfolgerungen zur Beschäftigungslage, zur Festlegung der strategischen Leitlinien für die legislative und operative Programmplanung im Raum der Freiheit, der Sicherheit und des Rechts, zur Festle-

16 Vgl. Europäisches Parlament: Entwurf einer Stellungnahme des Ausschusses für Internationalen Handel für den Ausschuss für konstitutionelle Fragen zu dem Vertrag zur Änderung des Vertrags über die Europäische Union und des Vertrags zur Gründung der Europäischen Gemeinschaft, EP-Dok. Nr. 2007/2286(INI), 4. Dezember 2007. 
gung der strategischen Interessen und Ziele der Union, sowie zur Verabschiedung von Beschlüssen über andere Bereiche des außenpolitischen Handelns der Union, die Beziehungen der Union zu einem Land oder einer Region, oder zu Fragen mit verteidigungspolitischen Bezügen, und zur Einschätzung der Bedrohungen, denen die Union ausgesetzt ist.

Wahl-, Benennungs- und Abberufungsrechte macht der Europäische Rat künftig geltend bei der Wahl seines Präsidenten und seiner vorzeitigen Entpflichtung, der Benennung des Präsidenten der Europäischen Kommission, der Ernennung (nach Zustimmung des Europäischen Parlaments und des Kommissionspräsidenten) und Abberufung des HVU-ASP.

In ihren Wirkungen nicht eindeutig definierte Beschluss- und Weisungsrechte macht der Europäische Rat gegenüber dem Ministerrat zur Bestimmung der strategischen Interessen der Union und zur Festlegung der Ziele ihrer Gemeinsamen Außen- und Sicherheitspolitik geltend. Darüber hinaus verfügt der Europäische Rat ab 2009 über ein Konsultationsrecht gegenüber jedem Mitgliedstaat, wenn dieser auf internationaler Ebene tätig wird oder eine Verpflichtung eingeht.

\section{Legitimität und reale Macht}

Diese Stärkung des Europäischen Rates im interinstitutionellen Gefüge der Union wurde seit Mitte der 1990er Jahre von den größeren EU-Mitgliedstaaten mit viel Einsatz und letztlich erfolgreich vorangetrieben. Sowohl deutsch-französische als auch spanisch-italienische und verschiedene britische Initiativen haben hierzu entscheidend beigetragen. Im Ergebnis wird über die Aufgabenzuweisungen des Europäischen Rates in den Bestimmungen des den EG-Vertrag künftig ersetzenden ,Vertrages über die Arbeitsweise der Europäischen Union ${ }^{\text {* }}$ (AEUV) ein Organ ins Leben gerufen, dessen Zuständigkeiten nicht nur wie bislang rein impulsgebender Natur sind. Die institutionelle Balance zwischen dem Europäischen Parlament, dem Rat und der Kommission wird dabei zu Lasten der Kommission und des Parlaments verändert.

Erst die genannten, neuen Handlungsermächtigungen des Europäischen Rates geben das sachliche Aufgabenspektrum und Potenzial des künftigen Präsidenten des Europäischen Rates wieder. Fraglich ist hierbei aber gerade angesichts der Funktionsbilanz des deutschen EU-Vorsitzes zweierlei:

Erstens steht zur Debatte, ob der künftige Präsident oder die Präsidentin über ausreichende personelle, administrative, finanzielle und politische Ressourcen verfügen wird, um Führungs-, Leitungs-, Vorbereitungs-, Kontinuitätssicherungs-, Konsensförderungs- und Vertretungsaufgaben gerecht zu werden, wie sie gegenwärtig von den Staats- und Regierungschefs der jeweils vorsitzführenden Länder wahrgenommen werden.

So drängt sich die Frage auf, ob der neue Präsident eine ähnliche Impulsgeberkraft und Repräsentationsleistung entwickeln kann wie sie vereinzelt von den heutigen Staats- und Regierungschefs in ihrer Funktion als Ratspräsident/in ausgeübt wird. Das strategische Agieren der Ratspräsidentin Merkel, die Dramaturgie der Präsidentschaft und ihres administrativen Unterbaus im Hinblick auf die Zuspitzung von Problemlagen und deren feierlich verkündete ,Lösung' auf zwei Europäischen Ratsgipfeln hatte entscheidenden Einfluss auf die EU- und G-8-Entscheidungen in der Klimapolitik oder die Entwicklung hin zu einem transatlantischen Wirtschaftsraum - allerdings unter Nutzung vielfältiger nationaler Ressourcen des deutschen Vorsitzes wie die guten Beziehungen zu den Vereinigten Staaten. Wie wird der Präsident des Europäischen Rates diese Ressourcen aufbauen und ihren Mangel kompensieren?

Ebenso zentral war die Vermittlungsrolle Merkels in den Verhandlungen über die Änderungen der europäischen Verträge. Welche Funktion werden Staats- und Regierungschefs in 
Zukunft in solchen Verhandlungen einnehmen? Werden Sie als Schattenpräsidenten weiterhin die Fäden in der Hand halten, an der Seite des Präsidenten des Europäischen Rates agieren oder ihre zentrale Rolle an ihn abtreten?

Verloren geht im neuen System auch die Repräsentationsrolle der derzeitigen EU-Ratspräsidenten gegenüber ihren nationalen Öffentlichkeiten. Die deutsche EU-Ratpräsidentschaft diente der Bundesregierung nicht nur zur Profilierung auf der europäischen Ebene, sondern eben auch auf der nationalen.

Die derzeitige herausgehobene Rolle der Regierungschefs als EU-Ratspräsidenten, die in dem ab 2009 gültigen System nicht kompensiert wird, macht die Entwicklung gewisser Antagonismen zwischen den nunmehr auch während ihrer eigenen Präsidentschaft zu ,normalen' Mitgliedern des Europäischen Rates degradierten Regierungschefs und dem neuen Präsidenten wahrscheinlich. Angesichts ihres möglichen Bedeutungsverlustes scheint der Anreiz der Staats- und Regierungschefs, den neuen Vorsitzenden des Europäischen Rates mit umfassenden Ressourcen und Aufgaben auszustatten und das Amt mit einer starken Persönlichkeit zu besetzen, nicht sonderlich groß. Auf die Beantwortung dieser Grauzonen des neuen Vertrages sollte sich die praktische Europapolitik rasch und konsequent einstellen.

Zweitens ist fraglich, ob der neue Präsident oder die neue Präsidentin des Europäischen Rates aufgrund des umfänglichen Aufgabenzuschnitts des Europäischen Rates über ein ausreichendes Maß an Legitimität verfügen wird. Denn während die auf sechs Monate ernannte EU-Ratspräsidentin Merkel dem Deutschen Bundestag gegenüber rechenschaftspflichtig war, gilt für den künftigen, gewählten EU-Vorsitz nichts Entsprechendes. Weder die nationalen Parlamente noch das Europäische Parlament verfügen über irgendwie geartete Instrumente, um regelaverses Verhalten des Präsidenten des Europäischen Rates zu sanktionieren.

Diese weitgehenden Befugnisse des Europäischen Rates und seines Vorsitzenden sollten einer baldigen Überprüfung und gegebenenfalls einer klaren Begrenzung unterzogen werden. Dies kann erstens durch die Einführung von Anhörungs- oder weitergehenden Kontroll- und Mitwirkungsrechten des Europäischen Parlaments erreicht werden, vor allem in denjenigen Fällen, in denen der Europäische Rat über vertragliche Beschlussfassungsrechte verfügt, die mittelbare Auswirkungen auf die Gesetzgebung der Union haben. Zweitens sollten sanktionsbewährte, selbstständige Anhörungs-, Zitier-, Frage- oder Interpellationsrechte des Europäischen Parlaments gegenüber dem Präsidenten des Europäischen Rates eingeführt werden, um die parlamentarische Legitimation dieses neuen Instituts europäischer Einigungspolitik abzusichern.

\section{Organisationsprobleme der Zukunft}

Außerdem ist in der europäischen Gesetzgebung - angesichts der in den letzten Jahren beobachteten Realentwicklung des Europäischen Rates im Verhältnis zu den anderen Fachräten - anzunehmen, dass sich seine Rolle als höchste Schlichtungs- und Schiedsinstanz in denjenigen Fällen weiterentwickeln wird, in denen mehrere Fachratsformationen zu gegensätzlichen Haltungen und Positionen im Gesetzgebungsprozess gelangen, und in denen der Allgemeine Rat nicht zu einer Einigung kommt. Der Europäische Rat wird sich in diesem Fall zu einer Art ,Oberrat' entwickeln, der nach dem Lissabonner Vertrag gemäß Artikel 15 Absatz 1 EUV zwar nicht ,gesetzgeberisch tätig“ wird, aber doch als letzte Instanz politische Beschlüsse verabschiedet und diese faktisch als Weisungen an die einzelnen Fachratsformationen weiterleitet. Diese Entwicklung wird dann aber auch Konsequenzen für die innerstaatliche Koordinierung der Europapolitik, die demokratische Kontrolle und die Vorbereitung und Durchführung künftiger Präsidentschaften in den Fachräten nach sich ziehen: 
Tendenziell wird der Druck auf die Staats- und Regierungschefs sowie die ihnen angeschlossenen Verwaltungsapparate zunehmen, entsprechende Koordinierungs- und Weisungsstrukturen aufzubauen beziehungsweise weiterzuentwickeln. Diese Entwicklung hat mittelbare Folgen für das Verhältnis der Außenminister (als Vertreter im Allgemeinen Rat) zu den Fachministern (als Vertreter in den Fachräten) sowie für die Einrichtung spezifischer Konsultations- und Koordinierungsmechanismen zwischen den ,national' geführten Fachpräsidentschaften und den Gipfelvorsitzen des Präsidenten des Europäischen Rates. Unklar ist nämlich, wer in längerfristig angelegten, strategischen Projekten wie der Energie- und Klimapolitik die Fäden zwischen Fach- und Gipfeltreffen zusammenführt, wer die heute üblichen ,Schlussfolgerungen des Vorsitzes ‘ künftig nicht nur formal autorisiert, sondern auch gegenüber Dritten im Sinne des Präsidenten des Europäischen Rates glaubwürdig vertritt. Werden sich Staats- und Regierungschefs hinter, ihren' Präsidenten stellen und sich selbst auch in denjenigen Feldern zurücknehmen, in denen die Versuchung nationaler ,Nebenpräsidentschaften" groß bleibt, sei es in der Außenpolitik oder in national bedeutsamen Themenfeldern? Oder werden sie auf die weiterhin bestehende Option ausweichen, häufiger ,normale ' Fachratssitzungen in Zusammensetzung der Staats- und Regierungschefs abzuhalten, um ihren Fachpräsidentschaften Profil und Prestige zu verleihen? Und damit dann aber auch den Präsidenten des Europäischen Rates faktisch zu demontieren? Und auf wen stützt sich der Präsident innerhalb des Generalsekretariats? Wird ihm ein neuer Dienst zugeordnet oder kann er auf alle bestehenden Generaldirektionen des Sekretariats zurückgreifen? Während für den ersten Fall bereits heute Vorkehrungen im Hinblick auf die Personalstruktur und -rekrutierung zu treffen wären, ist für den letzten Fall ein Mechanismus zu schaffen, der Konflikte um Zugriffe auf Personal und Finanzen mit dem Generalsekretär des Rates sowie dem HVU-ASP kanalisiert.

Konsequenzen sind auch für die innerstaatliche Strukturierung des Verhältnisses zwischen den nationalen Parlamenten und ihren Regierungen zu erwarten. Denn je nach Ausgestaltung der Beziehungen zwischen Regierung und Parlament in EU-Angelegenheiten werden sich Kooperations-, Kontroll- und Konfliktstrukturen verändern.

Außenpolitisch kann die Stärkung des Europäischen Rates im positiven Fall, durch die gemeinsame Positionierung der Staats- und Regierungschefs gegenüber Drittstaaten zu einer Stärkung der Union insgesamt führen. Demgegenüber bleibt allerdings die Gefahr einer Blockade des Europäischen Rates als politisch bedeutendstes Entscheidungsgremium der Staaten weiterhin bestehen, was dann zu einem Ausweichen einzelner Staatengruppen auf die , verstärkte Zusammenarbeit' führen kann. Diese Option birgt im Endeffekt die Gefahr der Aushöhlung der Union auf rein wirtschaftliche Zusammenhänge.

Entscheidend für das Funktionieren des neuen Systems im Europäischen Rat wird somit sein, welche Rolle die Mitglieder des Europäischen Rates dem Präsidenten im Alltag seiner Arbeit zugestehen wollen. Da er kein einzelstaatliches Amt innehaben darf, das heißt ihm eine direkte Hausmacht fehlt, kann er - und hier lässt der Vertrag von Lissabon vieles offen - zum Spielball der Staats- und Regierungschefs im Europäischen Rat werden, genauso aber aufgrund seiner Persönlichkeit eine starke Rolle gegenüber allen Organen der Europäischen Union spielen, oder sich genötigt sehen, seine Stärke aus der Zusammenarbeit mit den anderen europäischen Institutionen zu beziehen. 